\title{
Macular buckle with Morin-Devin T implant for pathological myopia with macular hole
}

\author{
Abhishek Anand ${ }^{1}$, Lalit Agarwal ${ }^{2}$, Nisha Agrawal ${ }^{3}$, Atul K. Anand ${ }^{1}$ \\ 'Vitreoretina Department, All India Institute of Medical Sciences, Patna, India; \\ ${ }^{2}$ Vitreoretina Department, Biratnagar Eye Hospital, Biratnagar, Nepal; \\ ${ }^{3}$ Cataract Department, Biratnagar Eye Hospital, Biratnagar, Nepal
}

\begin{abstract}
Introduction: Pathological myopia is commonly associated with myopic traction maculopathy, which includes foveoschisis, foveal retinal detachment, macular hole $(\mathrm{MH})$ and/or macular detachment (MD). Macular buckling is a rarely practiced extraocular surgical modality these days. The purpose of this study was to investigate the efficacy of primary buckling with Morin-Devin T implant for MD with MH and posterior staphyloma.

Case description: A 52-year-old female presented with light perception vision in her right eye with posterior staphyloma, localized neurosensory detachment, and $\mathrm{MH}$. She underwent primary macular buckling with Morin-Devin T implant. During the immediate postoperative day the wedge indentation was found misaligned to the fovea. A revision surgery was done after 2 weeks for repositioning of the macular wedge. Spectral domain optical coherence tomography confirmed indentation at the $\mathrm{MH}$ with resolution of subretinal fluid and hole closure. Her BCVA was 2/60 at 3 months postoperative and it remained the same even at 6 months of follow-up.

Conclusions: Primary macular buckling can be an effective procedure in eyes with $\mathrm{MH}$ with detachment and posterior staphyloma with or without associated foveoschisis. Morin-Devin T implant placement is a relatively simple procedure with short surgical time and excellent outcome.
\end{abstract}

Keywords: macular buckling, Morin-Devin T implant, myopic foveoschisis

\section{Introduction}

Pathological myopia with associated myopic traction maculopathy (MTM) is a relatively common posterior segment disease in the Asian population. ${ }^{1}$ MTM includes foveoschisis, foveal retinal detachment, lamellar or fullthickness macular hole $(\mathrm{MH})$, and/or macular detachment $(\mathrm{MD})^{2}$ and is generally associated with a posterior staphyloma. ${ }^{3}$ OCT studies have shown frequent association of myopic foveoschisis with $\mathrm{MH}$ with or without retinal

Correspondence: Dr. Nisha Agrawal, Cataract Department, Biratnagar Eye Hospital, AtithiMarg, Rani, Biratnagar-17, Nepal.

E-mail: drnisha agr@hotmail.com 
detachment and the pathogenesis and management of the same has inspired much debate in the literature. ${ }^{4}$

Release of epiretinal traction by pars plana vitrectomy with or without internal limiting membrane (ILM) peeling with gas or silicon oil tamponade has enjoyed a reasonable success. However, pars plana vitrectomy (PPV) with ILM peeling in high myopic eyes is surgically challenging and is associated with a low rate of $\mathrm{MH}$ closure and frequent complications. ${ }^{5,6}$ Scleral wall modulation by scleral shortening or episcleral buckling has been reported with good anatomical and functional results. ${ }^{6,7}$

\section{Methods}

Morin-Devin T implant \{France Chirurgie Instrumentation ( $\mathrm{FCl}$ ), Paris, France\}: Morin-Devin T implant has two components: a 4-mm wide silicone band and a 7-mm solid macular wedge (Fig. 1A). The T-shaped macular buckle is created by threading the band through the solid silicone macular wedge's planoconvex end (Morin-Devin " $T$ "-shaped macular wedge). ${ }^{8}$ The horizontal line of the " $T$ " is formed by the solid silicon band and the vertical line of the " $T$ " is formed by the macular wedge symbolically (Fig. 2). Although the indenting wedge end of the macular buckle is slightly convex on the non-indenting side but due to huge mismatch of convexity of the indenting and the non-indenting side, it would be more apt to call it planoconvex.

\section{Surgical technique}

$180^{\circ}$ temporal limbal peritomy was performed. Superior (SR), inferior (IR), and lateral recti (LR), and inferior oblique (IO) muscles were carefully isolated and secured by anchoring sutures using muscle hooks. Care was taken to secure all muscle fibers during $\mathrm{IO}$ isolation.

The 4-mm solid silicon band was first passed under the IR, IO, and LR. After passing underneath the $L R$, in the plane between the LR and the SR the 4-mm band was threaded through the slit provided in the indenting head of the macular wedge creating the $\mathrm{T}$ (Figs. $1 \mathrm{~B}$ and 2 ).

The macular wedge indenting head along with the threaded silicon band was then negotiated under the LR. A long curved forceps holding the macular wedge tip was used to negotiate the macular wedge and to place the indentation of the planoconvex wedge under the detached $\mathrm{MH}$ carefully. Care must be taken so that the convex indenting face of the wedge faces the sclera while negotiating under the LR. Simultaneous indirect ophthalmoscopy with a +20 diopter lens was used to confirm the desired indentation. Care was taken while manipulating the 


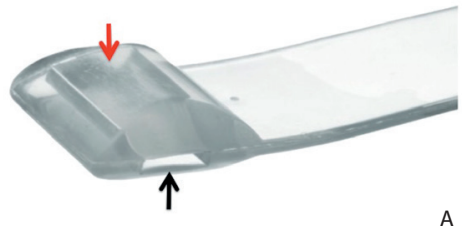

A

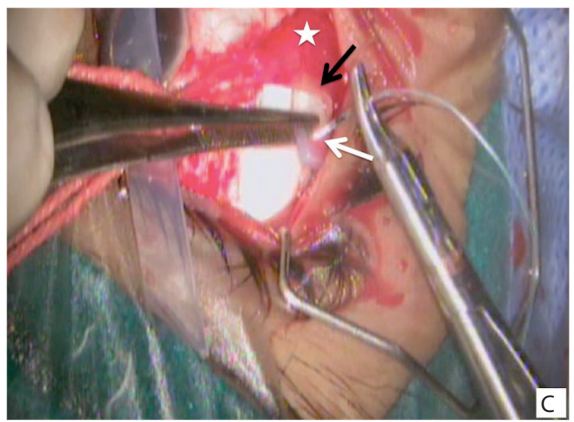

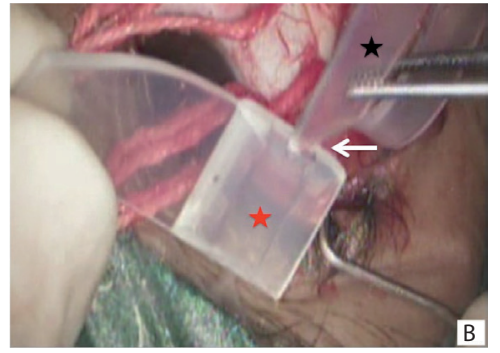

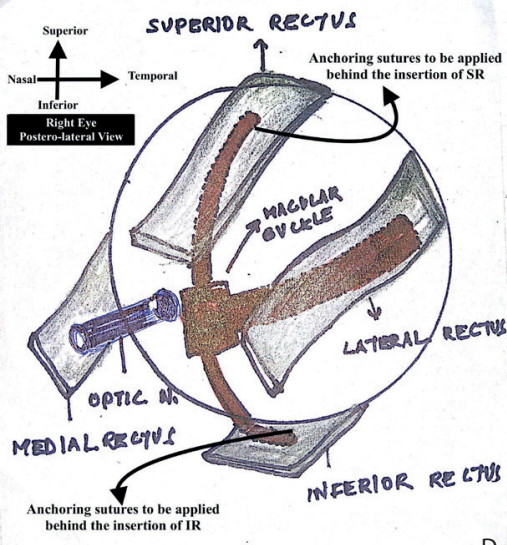

Fig. 1. Morin-Devin T Implant. (A) $7 \mathrm{~mm}$ solid macular wedge with biconvex end. "Red arrow" indicates the biconvex side of the macular edge, which will indent the macular area. "Black arrow" points to the slit in the solid macular wedge through which the silicon band will be threaded. (B) Creation of a T-shaped macular buckle by threading of a 4-mm silicone band through the solid silicone macular wedge's biconvex end (white arrow). "Black star" points to the solid 4-mm silicone band. "Red star" points to the solid macular wedge. (C) Anchoring of superior border of macular wedge with 5-0 Ethibond sutures to the sclera. "White star" indicates the lateral rectus (LR). Black arrow points to the superior border of the LR. White arrow points to the passing of the 5-0 Ethibond suture through the 7-mm macular wedge and being anchored to the sclera at the superior border of the LR. (D) Three-dimensional drawing of the right eye with Morin-Devin T implant from a posterolateral point of view. Free anterior ends of the 4-mm band secured just posteriorly beneath the insertion of the superior and inferior rectus, respectively. Free anterior end of the macular wedge secured to sclera just posterior and underneath the insertion of the LR. 


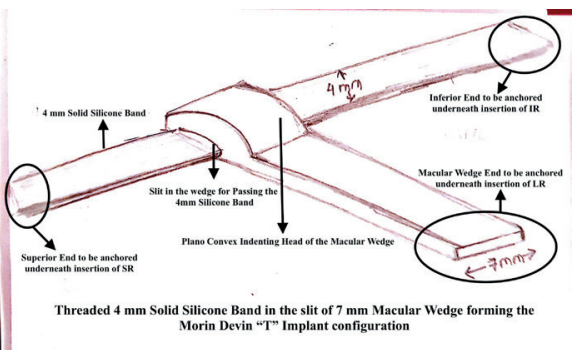

Fig. 2. Three-dimensional drawing of the final configuration of the solid silicone band and macular wedge after threading of the band into the slit provided at the indenting head of the wedge forming the "T" configuration.

wedge to the desired position so that inadvertent trauma to the optic nerve and short ciliary vessels could be avoided.

From our experience and from a literature search accurate positioning of the indenting head is very important. The indenting head once placed produces enough visible indentation at the macular area. The desired indentation to be achieved is the indenting bump should be slightly below the edge of staphylomatous excavation. Placement of the macular wedge is enough to achieve the desired indentation in most of the cases.

In cases of extreme staphyloma increasing the tension on the 4-mm solid silicone band can be used to increase the indentation.

The free anterior end of the macular wedge which formed the inferior end of the T was secured underneath the LR by anchoring its superior and inferior borders to the sclera with a 5-0 Ethibond suture. Three anchoring sutures were passed at the superior border and two at the inferior border of the 7-mm silicon macular wedge to prevent future displacement (Fig. 1C).

The $4 \mathrm{~mm}$ band was passed underneath the SR. The extra-long superior band was discarded and the cut end was secured nasal to the insertion of the SR with 5-0 Ethibond sutures. The inferior end of the 4-mm band was similarly fashioned and anchored nasal to the insertion of the IR. Tenon and conjunctiva were secured with a 8-0 polyglactin suture. The final position of the buckle with respect to the muscles is shown in Figure 1D.

In this case, only primary external macular buckling (PEMB) was performed. In cases of subrotal retinal detachment and especially detachment limited to the staphylomatous area or up to the vascular arcade, no drainage of subretinal fluid needs to be done. The macular fluid gets absorbed gradually. It may take few days to months for complete reabsorption of the fluid. In cases of extensive retinal detachment, subretinal fluid can be drained from a peripheral location. Air or gas tamponade can be used for the same. The available literature for PEMB has a success rate of around $90 \%$. 


\section{Case summary}

We report a case of a 52-year-old woman who had posterior staphyloma with $\mathrm{MH}$ and macular detachment limited to vascular arcade in the right eye (Fig. 3A). The patient underwent PEMB with Morin-Devin T implant as described above.

Postoperatively, however, the indentation of macular buckle was infero-temporal to the fovea. The patient underwent a revision buckling surgery after 2 weeks. Satisfactory results were obtained intraoperatively and postoperatively after the revision surgery (Fig. 3B).

At 3 months of follow-up, best corrected visual acuity (BCVA) improved to $2 / 60$. SD-OCT showed significant buckle effect and resolution of MD with hole closure (Fig. 3C). A persistent restriction of eye movement on lateral gaze was noted at the last follow-up. The visual acuity remained static even at 6 months of follow-up.

\section{Discussion}

MTM in varying severity invariably coexists with posterior staphyloma. Progressive axial elongation along with anteroposterior vitreous traction and taut ILM creates shearing forces responsible for foveoschisis with or without $\mathrm{MH}$ and retinal detatchment. ${ }^{2,9}$

Various authors have reported significant success of pars plana vitrectomy with ILM peeling and tamponade in MTM., ${ }^{5,10}$ Significant surgical expertise is required while working in an eye with abnormal scleral rigidity, longer axial length, mismatched instruments to axial length size, and visibly reduced contrast at the posterior pole due to myopic degeneration. Low $\mathrm{MH}$ closure rates, ${ }^{5}$ development of extrafoveal holes, ${ }^{6}$ progression of foveoschisis post ILM peeling ${ }^{11}$ and redetachment post tamponade removal ${ }^{6}$ mars its eventual success rate and its real success is questionable.

Macular buckling addresses the pathology by changing the configuration of the posterior pole from concave to planoconvex, thus relieving anteroposterior and tangential traction, scleroretinal mismatch and also reinforces the retinal
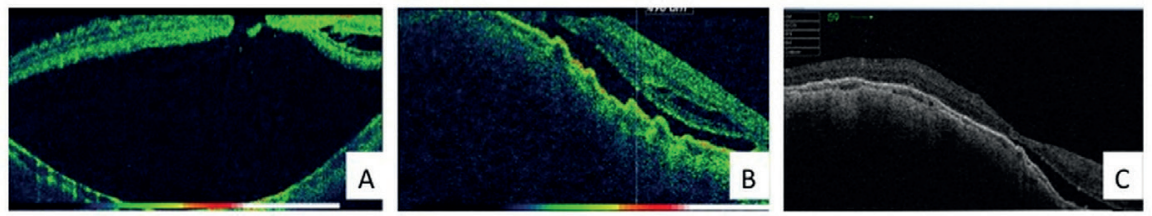

Fig. 3. SD-OCT showing reattachment after macular buckling with the Morin-Devin T implant. (A) Preoperative OCT showing macular hole with posterior ectasia of scleral wall. (B) One-month postoperative OCT showing persisting macular hole with significant indentation at the fovea. (C) Three-month follow-up showing closure of macular hole with persisting of detachment nasal to fovea. 
pigment epithelium and neurosensory retina adherence by bringing them together in a chorioretinal atrophied area.

Although eclipsed by PPV for a long time, macular buckling has shown resurgence in recent times with reports coming from various centers emphasizing high success rates of macular buckling with or without vitrectomy. ${ }^{6-8}$ In a case series reported by Ando F et al. comparing episcleral macular buckling (EMB) versus PPV in eyes with retinal detachment due to $\mathrm{MH}$ with posterior staphyloma, the retinal reattachment rate in EMB group was $93.3 \%$ after primary surgery and $100 \%$ after secondary surgery. In the PPV group, the retinal reattachment rate was $50 \%$ after primary surgery and $86 \%$ after secondary surgery using the EMB procedure, thus indicating a better anatomical success rate after primary EMB than after primary PPV. ${ }^{12}$ Many case series with primary EMB or EMB with PPV have reported higher anatomical and functional success rate over primary PPV alone..$^{6-13}$

Different macular buckles have been described in the literature. The most popular one is the Ando plombe explant (Ondeko Corporation Japan). It is a solid silicone rod with a metallic wire inside that allows it to be bent to obtain the desired buckling effect of the macular area. Mateo et al. coupled the indenting head to a 30-g optical fiber, which can be turned off and on, to help in accurate placement of the buckle by transillumination. ${ }^{14}$

Similar to the Ando plombe explant is the AJL macular buckle (AJL Ophthalmic Spain). ${ }^{14}$ This buckle is made up of PMMA material covered with silicone to increase its biocompatibility. It has a spherical indentation head while the Ando plombe has an ellipsoidal head. In order to get optimum indentation, the arm length and curvature in AJL is customized to the individual's eye. AJL can also be coupled with optical fiber for guided placement using transillumination. Both Ando plombe and AJL can be easily placed by just exposing the supero-temporal quadrant and thus scores over other macular buckles. ${ }^{7,14}$ However, indentation with the above buckles cannot be accurately titrated intraoperatively. The biggest deterrent is the cost of these explants in South Asia, which is almost 50-100 times more than the Morin-Devin T implant.

Another commonly used macular buckle is the adjustable macular buckle which has a solid silicone handle with a terminal plate for indentation. ${ }^{15}$ The terminal plate has two winglets on either side for passing mersilene suture. The two ends of the suture are circumnavigated and tied in the opposite quadrant under the medial rectus. The indentation can be increased or decreased by tightening or loosening the suture postoperatively. The main disadvantages are longer learning curve and requirement of LR disinsertion for securing the explant under LR. ${ }^{6,7}$

Some authors have also reported use of solid silicone sponge with or without a metal wire (L-shaped macular buckle and wire-strengthened sponge exoplant). ${ }^{15}$ However, long-term safety of the metal wire is not known and extrusion of sponge 
is a real concern from these explants. Also intraoperative and postoperative titration is not possible. Suturing of silicone tire and sponge by direct visualization of the staphylomatous area has been used in the past but requires disinsertion of muscles for macular exposure and there lies the risk of perforation of the extremely thin staphylomatous area. ${ }^{6,15}$

Complexity of different procedures and unfamiliarity has dissuaded macular buckling becoming popular, especially in the Indian subcontinent. The MorinDevin T implant scores a huge economic advantage. Unlike other procedures, the $T$ implant does not require muscle disinsertion, posterior suture, and any open surgical access to posterior pole making the procedure relatively simple. Inherent disadvantages of macular buckle like intraoperative risk of scleral perforation, compromise of short posterior ciliary circulation, damage to $\mathrm{ONH}$, abduction deficit, misalignment under the fovea, and late development of chorioretinal atrophy exist in T implants too. ${ }^{8}$

In our first attempt we had buckle misalignment. This can be prevented by using a customized curved forceps for placing the macular wedge, ability to differentiate false indentation produced by instrumentation, and better hand and eye coordination. Transillumination with an optical fiber has been used with other implants. ${ }^{14}$ No such commercially available modifications are available with the Morin-Devin T implant. An extrapolation can be done by using a retaining suture to fix a fiber-optic cable at the macular wedge which can be used to confirm the macular indentation and later removed by pulling off the fiber-optic cable. In a previous study, a $25-\mathrm{g}$ self-retaining chandelier endoilluminator along with a wide angle viewing system was used to confirm appropriate indentation. ${ }^{13}$

In our case, we achieved satisfactory chorioretinal apposition and closure of $\mathrm{MH}$ though with abduction deficit at 3-month follow-up on SD-OCT. We believe that MTM with coexisting pathology can be addressed with scleral wall reshaping using a relatively simple technique of macular buckling with the Morin-Devin T implant.

\section{References}

1. Wong YL, Saw SM. Epidemiology of pathologic myopia in Asia and worldwide. Asia Pac J Ophthalmol. 2016;5(6):394-402.

2. Johnson MW. Myopic traction maculopathy: pathogenic mechanisms and surgical treatment. Retina. 2012;32(Suppl 2):205-210.

3. Gaucher D, Haouchine B, Tadayoni R, et al. Long-term follow-up of high myopic foveoschisis: natural course and surgical outcome. Am J Ophthalmol. 2007;143:455-462.

4. Shimada N, Ohno-Matsui K, Baba T, Futagami S, Tokoro T, Mochizuki M. Natural course of macular retinoschisis in highly myopic eyes without macular hole or retinal detachment. Am J Ophthalmol. 2006;142:497-500. 
5. Ikuno Y, Sayanagi K, Oshima T, et al. Optical coherence tomographic findings in macular holes and retinal detachment after vitrectomy in highly myopic eyes. Am J Ophthalmol. 2003;136:477-481.

6. Mateo C, Burés-Jelstrup A, Navarro R, Corcóstegui B. Macular buckling for eyes with myopic foveoschisis secondary to posterior staphyloma. Retina. 2012;32(6):1121-1128.

7. Baba T, Tanaka S, Maesawa A, Teramatsu T, Noda Y, Yamamoto S. Scleral buckling with macular plombe for eyes with myopic macular retinoschisis and retinal detachment without macular hole. Am J Ophthalmol. 2006;142:483-487.

8. Devin F, Tsui I, Morin B, Duprat JP, Hubschman JP. T-shaped scleral buckle for macular detachments in high myopes. Retina. 2011;31(1):177-180.

9. Takano M, Kishi S. Foveal retinoschisis and retinal detachment in severely myopic eyes with posterior staphyloma. Am J Ophthalmol. 1999;128:472-476.

10. Kanda S, Uemura A, Sakamoto Y, Kita H. Vitrectomy with internal limiting membrane peeling for macular retinoschisis and retinal detachment without macular hole in highly myopic eyes. Am J Ophthalmol. 2003;136:177-180.

11. Shukla D, Dhawan A. Foveoschisis after vitrectomy for myopic macular hole with secondary retinal detachment. Eye. 2009;23:2124-2125.

12. Ando F, Ohba N, Touura K, Hirose H. Anatomical and visual outcomes after episcleral macular buckling compared with those after pars plana vitrectomy for retinal detachment caused by macular hole in highly myopic eyes. Retina. 2007;27:37-44.

13. Bedda AM, Hadi AMA, Lolah M, Shafy MSAA. A new sutureless illuminated macular buckle designed for myopic macular hole retinal detachment. J Ophthalmol. 2017;Article ID 6742164:7.

14. Mateo C, Medeiros MD, Alkabes M, Jelstrup AB, Postorina M, Corcostegui B. Illuminated Ando plombe for optimal positioning in highly myopic eyes with vitreoretinal diseases secondary to posterior staphyloma. JAMA Ophthalmol. 2013;131(10):1359-1362.

15. Susvar P, Vinay KS. Macular buckle in myopia. Sci J Med Vis Red Foun. 2015;XXXIII:71-74. 